\title{
Monitoring skeletal muscle dynamics and modelling the nonlinear response
}

\author{
M. Zakir Hossain ${ }^{1,2,3} \cdot$ Julian Grill ${ }^{2}$. Wolfgang Grill ${ }^{1,2}$ (I)
}

Received: 2 July 2019 / Accepted: 22 October 2019 / Published online: 4 November 2019

(c) Springer Nature Switzerland AG 2019

\begin{abstract}
A custom ultrasonic calliper was employed to monitor voluntary and externally excited muscle dynamics with synchronous electromyography. The activation, hold, and relaxation phases of the gastrocnemius muscle were monitored for maximum voluntary isometric contraction. Muscle belly shortening occurred during contraction and a post-contractile overshoot (lengthening) and subsequent exponential recovery of muscle dimension to the baseline were observed. Both the overshoot and recovery are attributed to the muscle as suggested by combined monitoring including electromyography and modelling with a lumped mechanical circuit containing idealized elements, such as a bidirectional linear motor unit, a ratchet, dampers, and springs. The rapid contraction and relaxation phases require a high-order filter or alternatively a kernel filter, attributed to the nervous system as suggested by external electric stimulation, which resulted in faster rise and relaxation times. The respective response function is modelled with an electrical lumped circuit. Together with empirically adjusted reaction times and corrections for droop in the hold phase, the monitored response is represented in close approximation by the combined electrical and mechanical lumped circuits. The refined combinatory model includes a ratchet as a novel nonlinear mechanical element. In combination with determined model parameters, it provides a refined evaluation scheme capable to model monitored muscle dynamics in physical activity in close approximation.
\end{abstract}

Keywords Ultrasonic monitoring of muscle dynamics - Modelling of muscle dynamics - Motor-spring-damper-ratchet lumped circuit $\cdot$ Muscle response to external stimuli $\cdot$ Post-tetanus autonomous contraction

\section{Introduction}

Skeletal muscle dynamics have been an intriguing field of study for centuries, having already been reported in 1667 $[5,18,25]$. Lumped circuit modelling of muscle dynamics was introduced by Hill [8]. This model illustrated in Fig. 1a and its adaptations have frequently been used to study muscle properties, allowing advances in understanding of fundamental dynamic properties. Recently more complex models have been used in an attempt to model properties not suitably represented by the basic Hill model, like the one proposed by Makssoud et al. [20] illustrated in
Fig. 1b. Hill's historical three-element muscle model $[7,8]$ has an active contractile element $\mathrm{E}$, which is a linear motor drive only capable of providing pull (shortening stress). It has two springs $S_{1}$ and $S_{2}$ with $S_{1}$ in series with the contractile element and $S_{2}$ parallel to both. These springs are considered capable of transmitting pull only, resisting elongation. Figure $1 \mathrm{~b}$ represents the model proposed by Makssoud et al. [20]. In addition to the classic contractile and elastic elements, it contains two mass-spring-damper arrangements. Both models connect to bones at each end (grey boxes). Further historical perspectives of modelling

Wolfgang Grill,wg@analogspeed.de | ${ }^{1}$ Faculty of Physics and Earth Sciences, University of Leipzig, Leipzig, Germany. ${ }^{2}$ ASI Analog Speed Instruments GmbH, Burgweg 8, 61460 Koenigstein im Taunus, Germany. ${ }^{3}$ Scientific Soccer School, Calgary, Canada. 


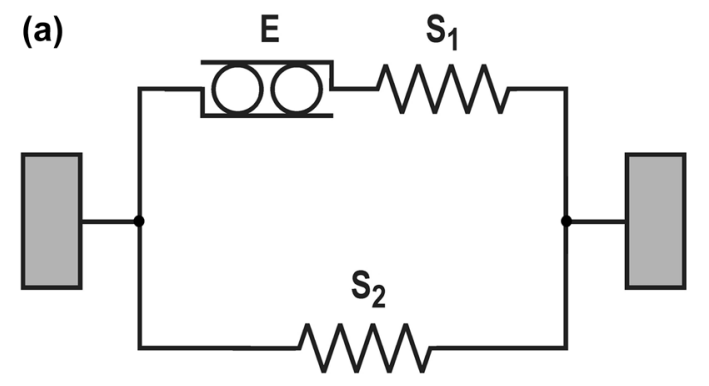

Fig. 1 a Actualized representation of A. V. Hill's three-elements muscle model with an active contractile element $E$, which is a linear motor drive only capable of providing pull in the historical arrangement. The springs $S_{1}$ and $S_{2}$ are arranged such that one is in series,

are provided in the detailed history of modelling of skeletal muscle dynamics described by Huijing [15].

In recent decades, it has become apparent that in vitro study of skeletal muscle does not adequately represent the in vivo situation. However, non-invasive monitoring of living skeletal muscle has been further refined, and applications are progressing. This approach also provides information on the dynamics of muscle contraction. Noninvasive monitoring includes ultrasonic imaging, near infrared spectroscopy, and electromyography (EMG). We have recently developed a high-resolution ultrasonic calliper (HRUC) for the non-invasive study of in vivo muscle dynamics [9-14]. This system is employed here for monitoring of the gastrocnemius muscle (GM) of human subjects in vivo.

This HRUC has revealed a property of muscles that current muscle models cannot emulate. It has been observed that during a sustained isometric contraction the active muscle shortens while stretching the tendon. Then upon the following relaxation the muscle extends to a length longer than the initial length (overshoot) and then afterwards slowly recovers to the original length. Similar asymmetric property of gastrocnemius and biceps muscles is presented in our previous research works $[11,12]$. The purpose of this paper is to explore possible mechanisms for this novel observed behaviour. A new model capable of accounting for the slow recovery to the initial length is presented.

The observations presented here focus on muscle dynamics under isometric (fixed-end) conditions and modelling adapted to emulate the experimental results obtained; muscle elongation and subsequent restoration to the original length follow isometric contraction. In some respects, these after-effects of contraction are similar to those first described by Kohnstamm [18] and Salmon [25] thereafter named "Katatonusversuch" (catatonus experiment), respectively, "catatonus". A further refined

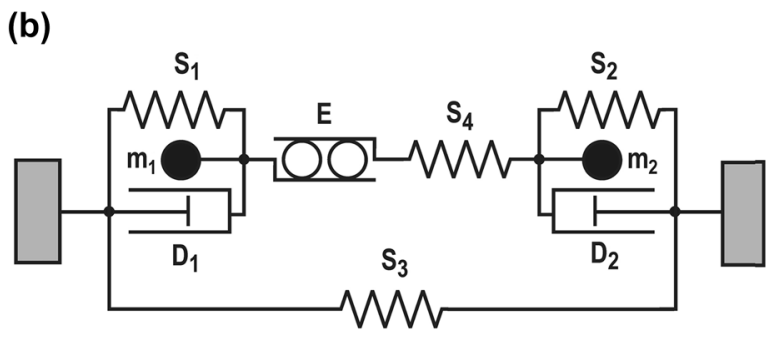

representing tendon, and one in parallel, allowing force development during passive stretch. $\mathbf{b}$ The model proposed by Makssoud et al. with two mass-spring-damper arrangements. Both models connect to bones (grey boxes) at both ends

discussion follows the presentation of the experiments and respective modelling.

Monitoring of the lateral expansion of muscle during contraction is performed by the observation of the timeof-flight (TOF) of ultrasonic waves travelling across the contracting and relaxing muscle. There is a small increase in the velocity of ultrasound under activation with respect to the relaxed muscle which is only $0.6 \%$ as demonstrated by Hossain et al. $[9,14]$. This variation of the velocity is sufficiently small that it can be ignored, and the time-of-flight can be used to estimate the length variations, assuming a fixed averaged velocity for the results presented here.

The mid-longitudinal area of whole GM muscle is confirmed constant by experimental observations [31]. The mid-longitudinal area remains constant over the length range ([19], chapter 2). Viscous properties are neglected as the monitoring is performed under the isometric conditions. The muscle is considered to be hyper-elastic, i.e. deformations are extremely large and strains are recoverable. Therefore no energy dissipation in a closed cycle of application and removal of stress [6]. This ensures the strain energy density function to be a function of strain. The stress can thus be calculated by differentiation of the strain energy density function with respect to the strain tensor [30].

The major goal of this study is the comparison of the observed elementary muscle dynamics with those predicted by a suitably developed mechanical model. A. V. Hill's classical mechanical model (Fig. 1a) is employed as a starting point for modelling. The technical elements lately utilized in mechanical lumped circuits for biomechanical modelling are usually idealized springs, viscous dampers (dashpots; [29], and force generators. Since neither the historical approach nor recently developed models were able to represent the experimental findings obtained here by HRUC monitoring, the modelling had to be adapted. 


\section{Materials and methods}

\subsection{Experimental procedures}

Ten healthy male subjects identified as soccer talents at the National Sports Institute of Bangladesh (BKSP) $13 \pm 1.25$ years of age with a body mass index (BMI) of $20.6 \pm 4.4$ (mean \pm SD) volunteered for this study. Inclusion criteria were that subjects had no recent lower extremity injury or pain at the time of monitoring. Informed written consent was obtained from BKSP's Ethics committee. Institutional and parents consents were received prior the experimental data collections. Data were collected in the presence of team coach and physical trainer. Every subject read and signed a consent form prior testing. Ultrasonic transducers were attached in opposing positions on the belly of the GM (Fig. 2a).

Surface electrodes for EMG were applied to the prepared skin. Two Kendall ARBO disposable surface EMG electrodes (10 mm) H135SG were used. One was placed on the monitored muscle belly for monopolar recording, and the reference electrode was placed on the Patella. Following amplification the EMG signal was recorded synchronously with the available second channel of the HRUC. The athletes were monitored in the seated position. The involved knee and ankle joints were kept at a $90^{\circ}$ flexed position (Fig. 3a).

The athletes were advised to initiate their effort when a ball was launched from a device in front of them. They were further instructed to pull their lower leg towards the back with all-out effort for as long as they could and end with a sudden release when they felt they could not maintain maximal effort. Contractions were isometric (fixed-end). Under the experimental conditions enforced by fixation of the lower leg (Fig. 3a), no joint angle change could be accomplished. Monitoring the GM belly diameter
Fig. 2 a Ultrasonic transducers were attached in opposing positions over the belly of the GM. b Simplified modelling of the muscle belly as cylinder
Fig. 3 a The subject reacts to an optical signal $(L)$, triggered electronically and initiated at zero time. $\mathbf{b}$ Variation of the length of the monitored muscle derived from the data obtained with the acoustic calliper. The letters relate to: $T$ : total reaction time, $C$ : force development phase, $H$ : holding phase, $E$ : extension phase, and $R$ : recovery phase of the observed muscle dynamics
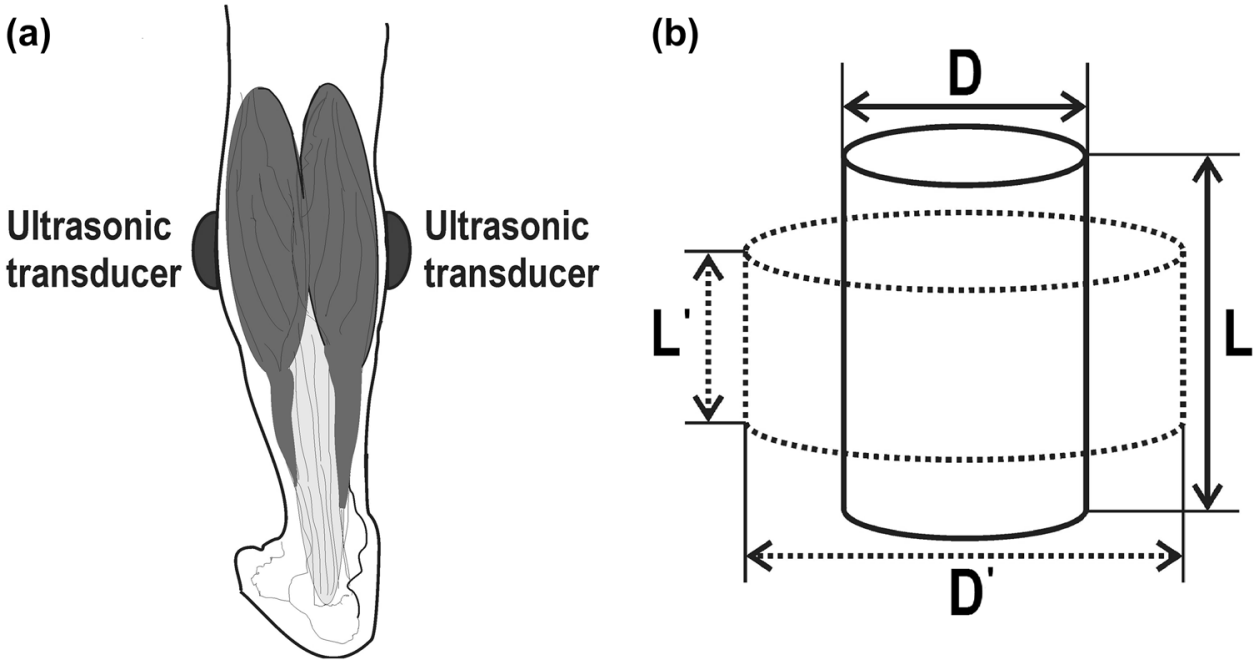

(a)

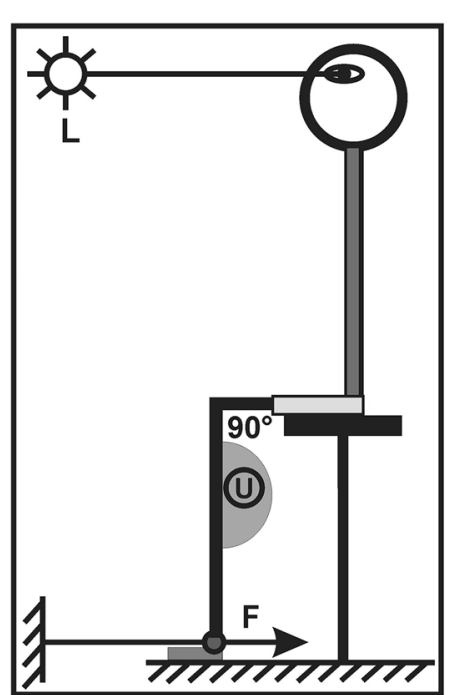

(b)

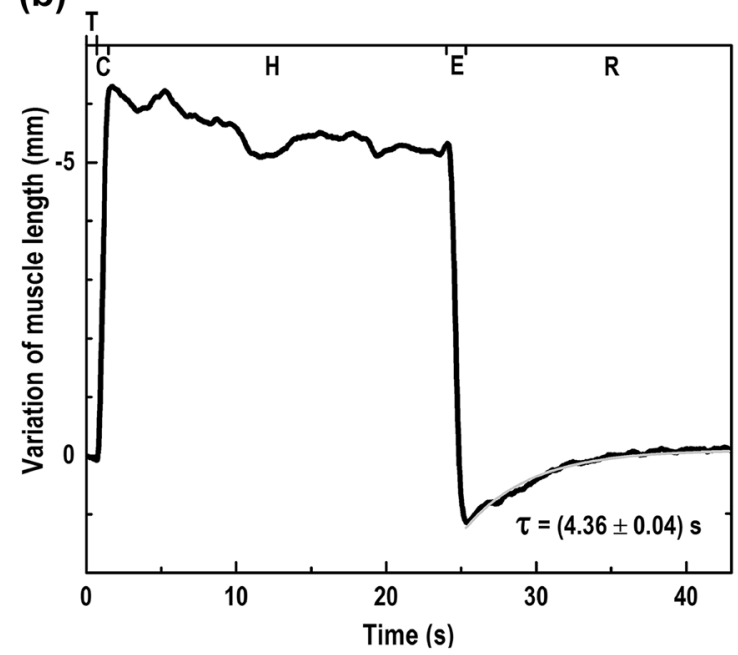


with the HRUC [13] was initiated with the release of the ball and continued for 30-50 s after the relaxation.

Additional procedures were followed to probe the nature of the droop, overshoot, and recovery of muscle belly diameter and corresponding estimated muscle length. In one instance, subjects were asked to hold their maximal effort for a specific duration (2-10 s) controlled with a visible signal (as shown in Fig. 3a), to determine if the overshoot and recovery were dependent on the duration of contraction. Maximal effort contractions lasting 2-7 s were also monitored with the ultrasonic transducers positioned orthogonal to the usual arrangement to see if orientation of the sensors affected the outcome (see Fig. 9 presented with the experimental results).

External electric stimulation $30 \mathrm{~mA}$ (symmetric bi-phasic supra-maximal stimulus of $762 \mu$ s duration) was used to avoid the neural control aspect of the response. This allowed quantification of the consequences of maximal activation of all motor units simultaneously. In addition, the neural processing time could be estimated. The time from initiation of the visual signal to the time of first muscle length change minus the time from initiation of the electrical signal to initiation of muscle length change would reveal this neural processing time. A healthy male subject with an age of 36 and $\mathrm{BMI}$ of 23.2 volunteered for this experiment. Muscle stimulating electrodes, adhesive $50 \mathrm{~mm}$ round surface electrodes (REF 282000, Schwa medico ${ }^{\circ}$ ), driven by an electronic stimulator (EMP 4 Pocket, Pierenkemper GmbH Wetzlar, Germany), were attached to the ends of the GM belly. Stimulation with 4-5 consecutive pulses was conducted on the right GM. The 4-5 consecutive pulses were used to compare the responses of the gastrocnemius muscle with the identical external electric stimulation. The average shortening, lengthening, and undershoot of 5.98, 6.94 , and $2.65 \mathrm{~mm}$ are observed respectively, and a typical response for a single pulse is shown in Fig. 12a.

\subsection{Data analysis}

Based on the assumption of lateral isotropy and conservation of volume $[2,4,16,17,23,24,27,28]$, the muscle length was derived from the lateral dimension of the muscle belly by geometric reasoning (Fig. 2b):

$\pi D^{2} / 4 L=\pi D^{\prime 2} / 4 L^{\prime}$

where $L, D$ and $L^{\prime}, D^{\prime}$ represent the initial and final muscle belly length and diameter, respectively, (Fig. 2). From this follows:

$L^{\prime} / L=D^{2} / D^{\prime 2}$

The underlying assumption of sufficient radial (lateral) isotropy of the muscle should be justified by the respective microscopic structure. This assumption was tested by monitoring in 2 orthogonal radial directions across the muscle.

The initial diameter $(D) 12.73 \mathrm{~cm}$ of the relaxed muscle belly was determined from the observed TOF of the chirped acoustic waves and the velocity of ultrasound of $1569 \mathrm{~m} / \mathrm{s}$, as previously confirmed for muscle [13]. For the initial muscle belly length $(L)$ of each athlete, a representative value of $15 \mathrm{~cm}$ was assumed. If necessary, this value could be determined with accuracy by medical imaging. The initial length is needed as a parameter for each subject to determine the length variation of the muscle belly under isometric contraction (Fig. 3b). Uncertainties concerning the determination of the length are expected to be less relevant than the influence of the simplification involved in the representation of the active part of the muscle as a cylinder, which is required as an intermediate step for modelling by a lumped mechanical circuit (Fig. 4). The monitored muscle response involves the reaction to a visual signal with the recording starting at time $t=0$, representing the time at which the optical signal is activated (Fig. 3a).

\section{Results and evaluations}

An example of data obtained from monitoring of the GM of an athlete is displayed in Fig. 3b. It shows the rapid shortening of the muscle on initiation of the contraction, a droop representing slight elongation over time then rapid elongation on relaxation when the athlete could no longer hold the maximal contraction. For each contraction, we measured the duration of the following phases: the delay between visual command and the onset of the muscle response (total reaction time $T$ ), the build-up of maximum voluntary isometric contraction (contraction phase C), maintained maximum voluntary isometric contraction (holding phase $H$ ), sudden withdrawal of effort (relaxation phase $E$ with overshoot), and finally the recovery phase $\mathrm{R}$ following the overshoot with exponential return to baseline. In addition, the peak rate of rise of force was determined by differentiation and the return to baseline was quantified by a time constant for the exponential shortening associated with this phenomenon.

\subsection{Mechanical model}

In the approach presented here (Fig. 4), the model is based on a linear drive, which is used instead of a force generator to achieve a closer approximation to the linear micromotor devices, action of actomyosin complex in sarcomeres, present in the actual muscle. In addition, springs and dampers are employed, as they are commonly utilized in lumped circuit modelling for biomechanical applications 
Fig. 4 a Developed lumped mechanical circuit with linear motor drive, springs, ratchet, and damper suitably arranged to represent the monitored muscle dynamics. b The elastic behaviour of the tendon modelled with an idealized spring and a hook, transmitting extensional stress only as needed in any application involving compressional stress of the tendon

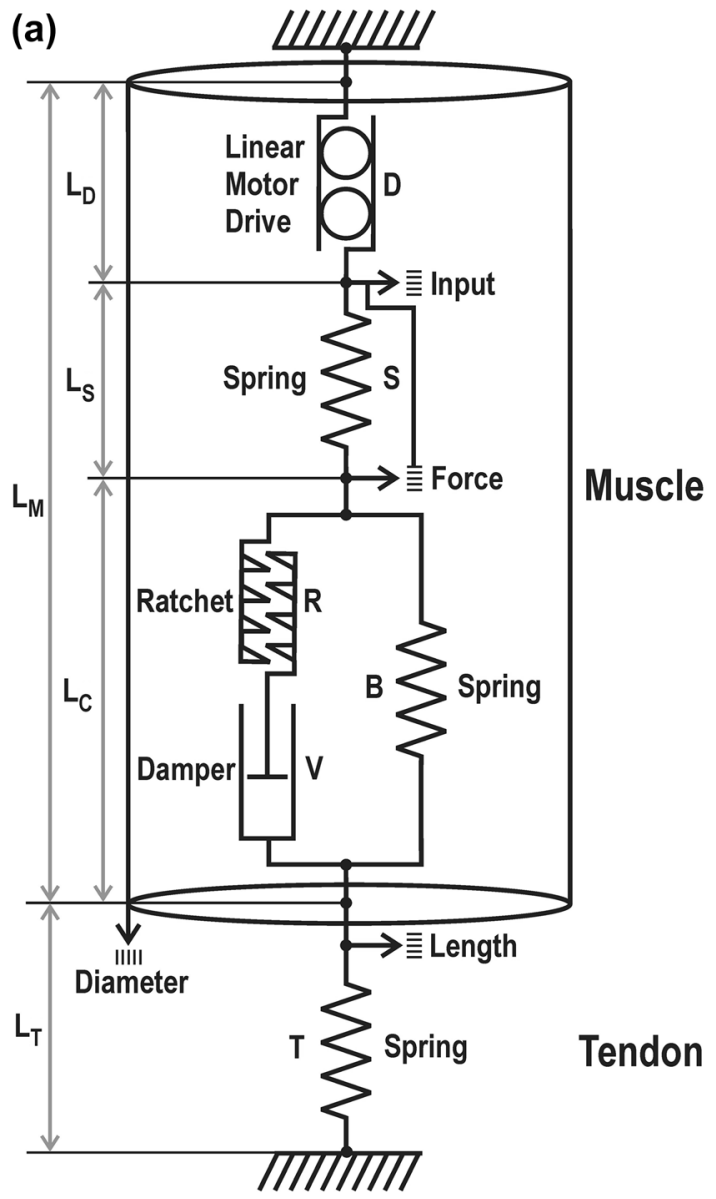

(b)

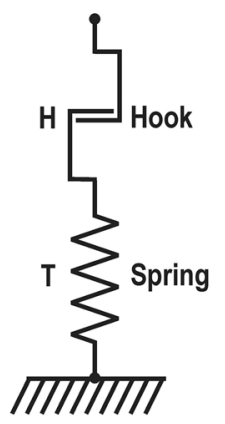

Tendon

To model the observed asymmetric nonlinear response, an idealized mechanical ratchet had to be introduced. This represents a refinement of the established modelling required to describe the experimental observations of the overshoot following the termination of muscle contraction and the subsequent recovery. The ratchet is mounted in series with a damper and both in parallel with a spring. Although ratchets have previously been employed in modelling, this has only been done to represent the motor mechanism [3, 22]. The current application here is in addition to the motor.

It should be mentioned that the special features implemented by A. V. Hill concerning the rubber like spring and contractile linear motor drive already indicate the need for non-symmetry and in today's terms nonlinear elements. Additionally, the implementation of a novel mechanical element, a ratchet, is required to explain the overshoot and subsequent recovery by modelling with a lumped mechanical circuit.

\subsection{Equations relating to modelling}

In accordance with Fig. 4 , the total length $L_{\text {tot }}$ of the gastrocnemius muscle (GM) and tendon, which is constant under isometric activities, can be expressed for all times tas: 
$L_{\text {tot }}=L_{M}(t)+L_{T}(t)=L_{D}(t)+L_{S}(t)+L_{C}(t)+L_{T}(t)$

Variations from each individual $L_{N}$ for the relaxed state where $F_{N}=0$ for all $N$ representing the different indices $C$, $D, M, S, T$ are denoted by $\Delta L_{N}$.

Each element of the mechanical lumped circuit can, respectively, transfer a push or pull relating to pairs of forces $+F_{N}$ and $-F_{N}$ aligned with the element. As suitable for the treatment of lumped circuits, where components with cross sections are modelled by lines, the stress is represented by $F_{N}$ only. Positive $F_{N}$ relates to pull and therefore tensile stresses for the respective element in the modelled muscle or tendon. An activated muscle will supply and be subject to a positive stress.

Springs $\mathrm{S}$ and B of the modelled muscle (Fig. 4a) would provide a so-called passive force under external elongation of the muscle. For internally provided forces caused by the linear motor drive in modelling, one of these springs, namely $B$, is essential for the observed and, respectively, modelled recovery phase (denoted by $R$ in Fig. $3 \mathrm{~b}$ ) following activation.

The idealized ratchet employed here is of the compressive type and only transmits compressive stress. The function of the ratchet in the lumped circuit can thus be represented by a Heaviside function $\mathrm{H}$ as defined by Abramowitz and Stegun [1]:

$H(x)=0$ for $x<0$ and $H(x)=1$ for $x^{3} 0$

Under the convention that tensile stresses relate to positive forces a time-dependent force $F_{1}$ acting on one end will be transferred to the other end by a compressive ratchet such that the force $F_{2}$ transferred is given by:

$F_{2}=F_{1} H\left(-F_{1} / U_{F}\right)$

with $U_{F}$ representing one unit of the force (one Newton according to S.I.). This is necessary here since $H$ can only operate on numbers. An alternative notation avoiding the Heaviside function can be based on:

$F_{2}=F_{1}\left(1-F_{1} / I F_{1} I\right) / 2$.

For the serial arrangement in the lumped mechanical circuit (Fig. 4) holds:

$F_{M}(t)=F_{D}(t)=F_{S}(t)=F_{C}(t)=F_{T}(t)=F(t)$

with $F(t)$ introduced to simplify the notation. Since:

$F_{C}(t)=F_{B}(t)+F_{R}(t) \quad$ and: $\quad F_{R}(t)=F_{V}(t) \quad$ follows:

$F_{C}(t)=F_{B}(t)+H\left(-F_{R}(t) / U_{F}\right) \eta \mathrm{d} / \mathrm{d} t \Delta L_{C}(t)$

with $\eta$ the damping coefficient characterizing the idealized viscous damper. Since

$F_{R}(t)=F_{C}(t)-F_{B}(t)$ and $F_{B}(t)=F_{S}(t)$ follows with the short notation:

$F_{l}(t)=F_{B}(t)-F_{S}(t):$

$F_{C}(t)=F_{B}(t)+H\left(F_{l}(t) / U_{F}\right) \eta \mathrm{d} / \mathrm{d} t \Delta L_{C}(t)$

The model presented here solely serves the purpose of describing isometric activities observed in this report. The model therefore contains no masses since kinetic forces were negligible.

The input causing the monitored and modelled response, also addressed as reaction, is the command. The input is represented by any normalized input function $J(t)$ varying between 0 for the command relaxed and 1 for maximum isometric contraction. Variations in length of any unit $N$ are here expressed as $\Delta L_{N}(t)$ and relate to strain. Positive $\Delta L_{N}(t)$ represents an elongation. For the respective variation of the length of the motor $L_{D}$, represented by $\Delta L_{D}(t)$ holds (as defined here):

$\Delta L_{D}(t)=\ell J(t)$

$\ell$ is a parameter of the model relating to amplification including the (negative) sign in regard to the command and respective motor action (shortening), specific for the muscle at the time of monitoring.

In addition to the motor other idealized elements serving the purpose of modelling the monitored response with a minimum of components are introduced in the lumped mechanical circuit. The spring $S$ introduced in the muscle changes length in proportion to the force generated by the motor drive. This simplifies any comparison of the here introduced lumped mechanical circuit driven by a linear motor element, as present in the real muscle, with already established lumped circuits employing idealized force generators. The spring $S$ introduced here is also needed to describe the mechanical properties inherent to the muscle. In established modelling, the spring $S$ is usually combined with the spring modelling the tendon, here denoted with $T$. Since $S$ and $T$ are connected in series this mainly leads to differences concerning the actual length of the muscle belly with respect to the tendon.

To relate to actual observations, the response modelled here is defined as the resulting time-dependent deviation $\Delta L_{M}(t)$ from the length of the muscle $L_{M}$ in the fully relaxed state. This variation of the muscle length according to the model (Fig. 4a) is given by:

$\Delta L_{M}(t)=\Delta L_{D}(t)+\Delta L_{S}(t)+\Delta L_{C}(t)=-\Delta L_{T}(t)$

For any of the idealized springs $N$ behaving according to Hooke's law such that stress, expressed here by $F_{N^{\prime}}$ is directly proportional to strain, the spring constant shall be $k_{N}$. It should be noted that the definition established for stress and strain leads to a different sign than the convention for Hooke's law involving forces and the path of the 
free end of the spring. For $\Delta L_{N}$ relating to strain therefore holds:

$\Delta L_{N}=F_{N} / k_{N}$ respectively: $F_{N}=k_{N} \Delta L_{N}$

The spring constant $k_{N}$ is a parameter of the model that has to be adjusted to the monitored muscle according to the observed response.

From Eqs. 5 and 6 and definition of the input function, the length of the muscle can be described as:

$\Delta L_{M}(t)=\ell J(t)+F(t) / k_{S}+\Delta L_{C}(t)=-F(t) / k_{T}$

Due to the influence of the ratchet (Eq. 4) and since according to Fig. 4 a $\Delta L_{B}(t)=\Delta L_{C}(t)$, the force $F(t)$ can be defined as:

$F(t)=H\left(F_{l}(t) / U_{F}\right) \eta \mathrm{d} / \mathrm{d} t\left(\Delta L_{C}(t)\right)+k_{B} \Delta L_{C}(t)$

Since

$\Delta L_{C}(t)=-\Delta L_{D}(t)-\Delta L_{S}(t)-\Delta L_{T}(t)$

the length variation of the combined $C$-element can be expressed as:

$\Delta L_{C}(t)=-\ell J(t)-F(t)\left(1 / k_{S}+1 / k_{T}\right)$

The force $F$ describing the stress experienced by this combined element is therefore:
For a better understanding, this can be separated into two cases. For case 1 with an inactive ratchet $(H(t)=0)$ holds:

$\Delta L_{M}(t)=-\left(1 /\left(k_{T}\left(1 / k_{B}+1 / k_{S}+1 / k_{T}\right)\right) \ell J(t)\right.$

As to be expected from a system with reacting springs only, this represents a simple proportional response of the type:

$\Delta L_{M}(t)=d J(t)$

with $d$ as the factor relating input and response. In this case, the individual $k_{N}$ characterizing the properties of the springs, cannot be derived from monitoring of $\Delta L_{M}(t)$ alone.

The combination of all springs into one spring, usually representing the tendon in historical modelling, is fully sufficient to describe the length of the tendon and to derive respective forces from the actual spring constant whenever dampers are absent or, respectively, disengaged by $H(t)=0$. Figure 5 demonstrates the response for this case representing an all elastic model according to Hooke's law. This reduced model cannot provide the overshoot observed following deactivation.

For the second case, valid during all times when the ratchet is engaged $(H(t)=1)$, the differential Eq. 10 can be written as:

$F(t)=H\left(F_{I}(t) / U_{F}\right) \eta \mathrm{d} / \mathrm{d} t\left(-\ell J(t)-F(t)\left(1 / k_{S}+1 / k_{T}\right)\right)-k_{B}\left(\ell J(t)+F(t)\left(1 / k_{S}+1 / k_{T}\right)\right)$

finally leading to:

$$
\begin{aligned}
0= & F(t) k_{B}\left(1 / k_{B}+1 / k_{S}+1 / k_{T}\right) \\
& -H\left(F_{l}(t) / U_{F}\right) \eta \mathrm{d} / \mathrm{d} t F(t)\left(1 / k_{S}+1 / k_{T}\right) \\
& +k_{B} \ell J(t)-H\left(F_{l}(t) / U_{F}\right) \eta \mathrm{d} / \mathrm{d} t(\ell J(t))
\end{aligned}
$$

With $\Delta L_{M}(t)=-F(t) / k_{T}$ and subsequently $F(t)=-k_{T} \Delta L_{M}(t)$ and with the Heaviside function reformulated in dependence of $\Delta L_{C}$ with $U_{L}$ as a unit of length follows:

$$
\begin{aligned}
& \left(k_{T} k_{S}+k_{B} k_{T}+k_{B} k_{S}\right) \Delta L_{M}(t)-\left(k_{S}+k_{T}\right) \eta \mathrm{d} / \mathrm{d} t\left(\Delta L_{M}(t)\right. \\
& \quad=-k_{B} k_{S} \ell J(t)+k_{S} \eta \mathrm{d} / \mathrm{d} t(\ell J(t))
\end{aligned}
$$

This Eq. (11) describes the response of an additive combination of a linear system with a first-order Bessel high-pass filter, that is achieved with a combination of a Kelvin-Voigt element and a spring (Hooke element), constituting a (first order) Poynting-Thomson model also

$$
\begin{aligned}
0= & -\Delta L_{M}(t) k_{T} k_{B}\left(1 / k_{B}+1 / k_{S}+1 / k_{T}\right)-H\left(\mathrm{~d} / \mathrm{d} t \Delta L_{C}(t) / U_{L}\right) \eta \mathrm{d} / \mathrm{d} t k_{T} \Delta L_{M}(t)\left(1 / k_{S}+1 / k_{T}\right) \\
& +k_{B} \ell J(t)-H\left(\mathrm{~d} / \mathrm{d} t \Delta L_{C}(t) / U_{L}\right) \eta \mathrm{d} / \mathrm{d} t(\ell J(t))
\end{aligned}
$$

Since $\Delta L_{C}(t)$ can be expressed as:

$$
\Delta L_{C}(t)=\left(1+k_{T} / k_{S}\right) \Delta L_{M}(t)-\ell J(t)
$$

the differential equation relating the monitored response concerning the length variation of the muscle $\Delta L_{M}(t)$ to the command (input function) $J(t)$ can finally be written as: addressed as Zener model of the Kelvin type, as shown in Fig. 6 . Since the damper is always engaged, the response exhibits overshoots for both activation and deactivation (at times 0 and 1 in the graph) and cannot describe the observed non-symmetric response.

$$
\begin{aligned}
& 0=\left(k_{T}+k_{B} k_{T} / k_{S}+k_{B}\right) \Delta L_{M}(t) \\
& \quad-H\left(\mathrm{~d} / \mathrm{d} t\left(\left(\left(1+k_{T} / k_{S}\right) \Delta L_{M}(t)-\ell J(t)\right) / U_{L}\right) \eta \mathrm{d} / \mathrm{d} t\left(\left(1+k_{T} / k_{S}\right) \Delta L_{M}(t)-\ell J(t)\right)\right. \\
& \quad+k_{B} \ell J(t)
\end{aligned}
$$


Fig. 5 a Modelling of the muscle as obtained from a reduced modification of the developed model (Fig. 4a) according to case 1. b Response (solid grey) of the reduced mechanical lumped circuit for normalized input (dotted) and the, respectively, derived and normalized response function. The time scale is normalized to the length of the desired action (a)

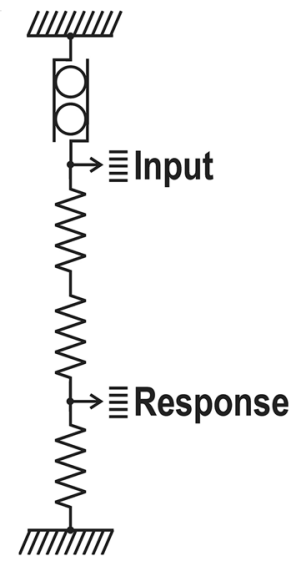

(b)

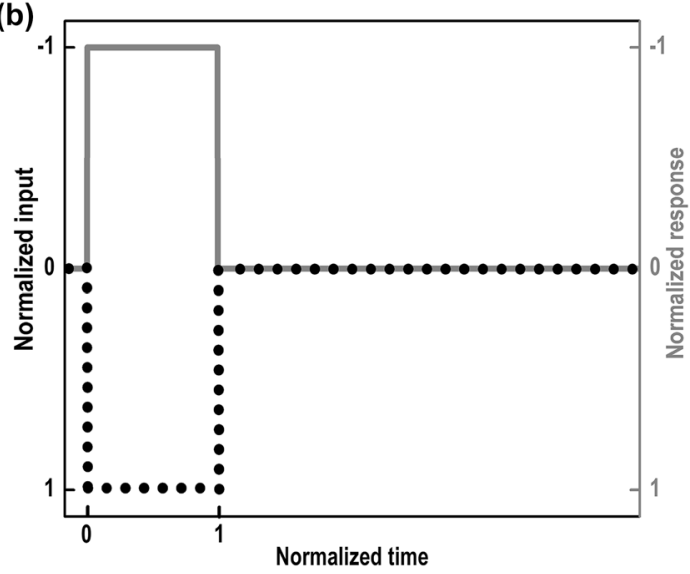

(a)

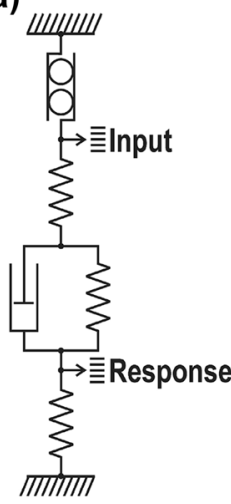

(b)

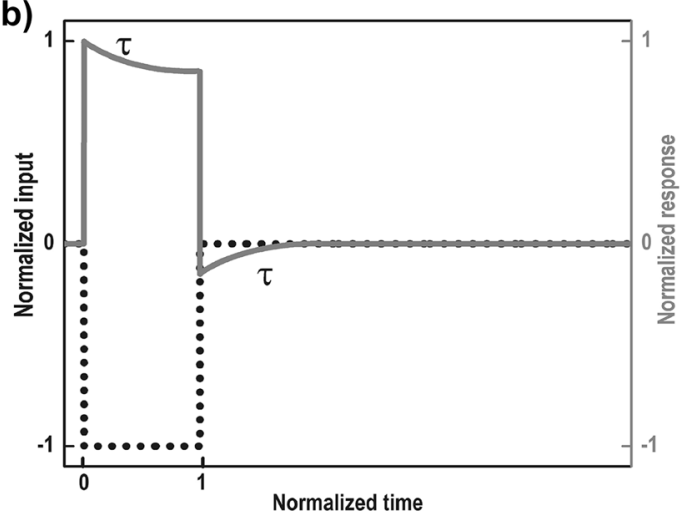

Fig. 6 a Simplified mechanical lumped circuit without ratchet with established models for comparison. b Graph with normalized input (dotted) and the derived normalized response (grey) for the simplified circuit

Fig. 7 a The mechanical lumped circuit with ratchet and damper. $\mathbf{b}$ The response (solid grey) generated by this system from Eq. 10 as derived for a normalized input (dotted) (a)

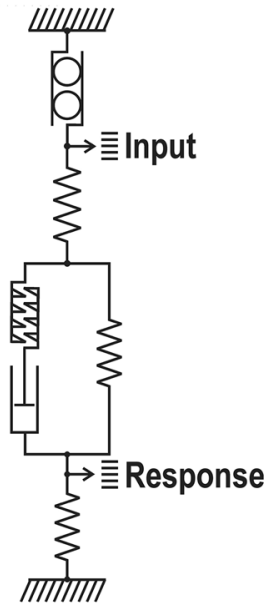

(b)

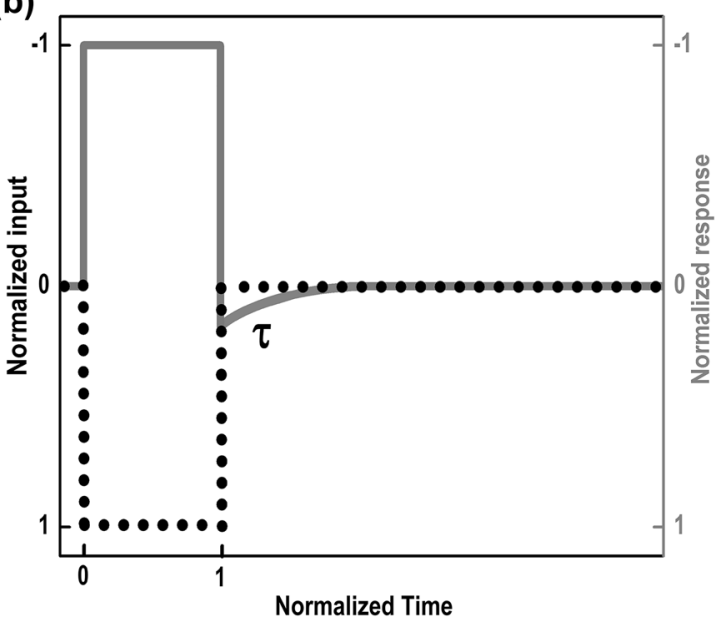

Since an overshoot is only observed for the deactivation, the damper needs to be activated and deactivated by the ratchet and a respective Heaviside function as expressed in Eq. 10, to obtain the type of response typically observed and displayed in Fig. 7. Due to the action of the ratchet, for normalized times $t<1$ resembling case 1 being active, the simplified muscle can be represented by bidirectional linear drive and spring elements only, whereas 
for all $t \geq 1$ the response of the muscle to the linear drive is presented by a Poynting-Thomson model.

The model presented in Fig. 7 achieves the overshoot following deactivation at normalized time 1 due to the ratchet. This is followed by an exponential recovery in close approximation to the experimental observations which is dependent on the properties of the damper. The combination of a ratchet and a damper in the model (Fig. 7) can also be interpreted as an idealized shock absorber with no resistance for dilatation and viscous damping for contraction. This equivalent alternative is of importance for the interpretation, since it avoids the features of a potentially infinite lengthening of the ratchet compensated by $a$, respectively, infinite shortening of the damper that could otherwise occur under cyclic motions. Such effects would of course only occur with idealized modelling components without length restrictions as commonly employed in lumped circuits.

\subsection{Comparison of experimental results and modelling}

The derived model Eq. 10 is solved with a suitable program based on MathCad7 ${ }^{\circ}$. For a normalized input, adjusted empirically to the reaction time, duration and droop of the hold phase, the amplification factor relating to the drive, the properties of the springs, and the damping constant are adjusted to fit the response to the observed muscle dynamics (Fig. 8).

The droop with irregular fluctuations observed during the holding phase is due to a reduction in the muscle activity either because of decreasing central command or because of intramuscular fatigue, neither of which can easily be described by modelling (compare Figs. 8,9 ). To address this, an adjusted linear droop is introduced to the input function as a modification.

Individual variability in the response can be programmed in the input function, including delay due to reaction time, hold time, and droop. One result is displayed in Fig. 8b. In Fig. 9a, the result observed for a better trained athlete exhibiting a more stable hold phase (less droop) is shown. The persistence of the overshoot in this circumstance would suggest that it is not a consequence of the droop. Also illustrated in Fig. $9 \mathrm{~b}$ are the results of ultrasonic monitoring across the tibia bone, observed in orthogonal direction with respect to all other results of monitoring presented here. This indicates that the observation of the overshoot and recovery cannot be attributed to anisotropic deformation in lateral directions.

The mechanical lumped circuit used for modelling does not include higher-order filters which would be needed to represent the region of activation and deactivation rounded off with steady first and higher-order derivatives as observed experimentally (Figs. 10a, 11).

Since much faster activation and deactivation phases were observed when contraction was elicited with external electric excitation of the muscle (see results presented in 3.5 below), the observed slower voluntary response was attributed to the nervous system. Therefore, electrical lumped circuits were introduced for modelling of the fast responses related to activation and deactivation.

\subsection{Electrical lumped circuit}

The reaction delay and especially the observed finite slope of the rise and fall of force, as well as soft curved onsets, and similarly observable rounded off ends of rapid movements are all attributed to brain processing and subsequent signal transmission in the nerves. For the purpose
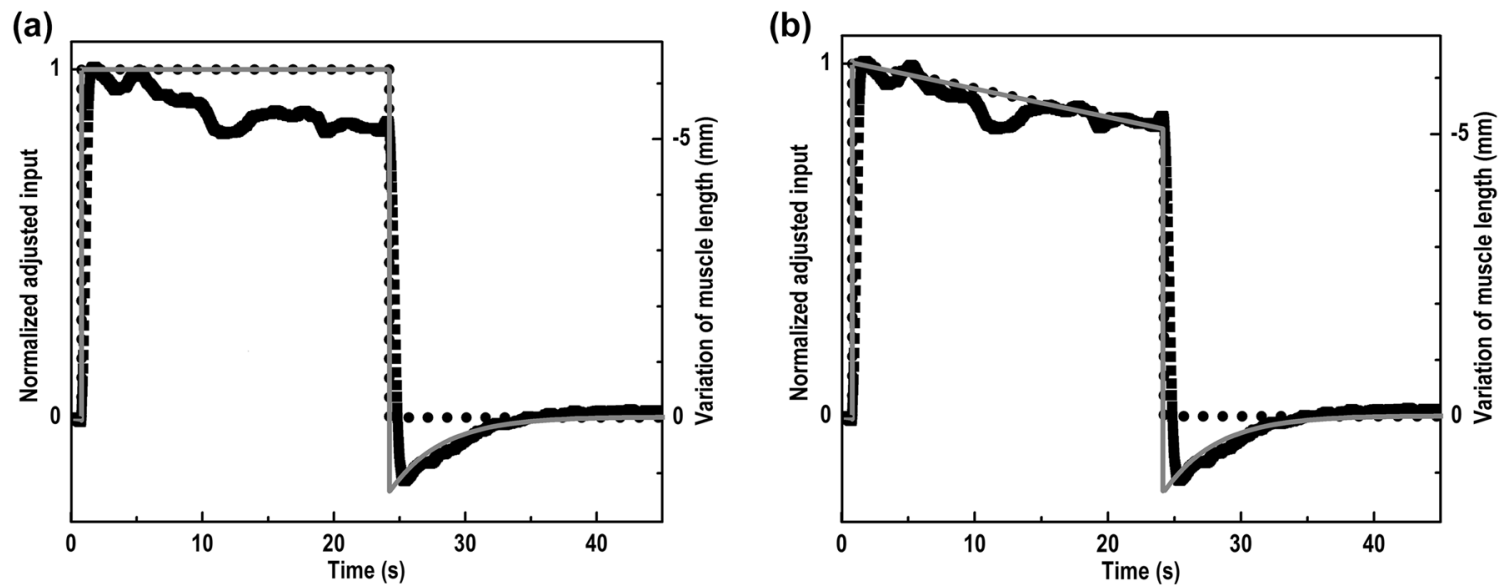

Fig. 8 a The normalized input (dotted) and modelled output (grey) with suitably adjusted parameters to fit the observed muscle response (black). b The input (dotted) and the measured (black) and modelled response (grey) for an input function with an adjusted linear droop 
(a)

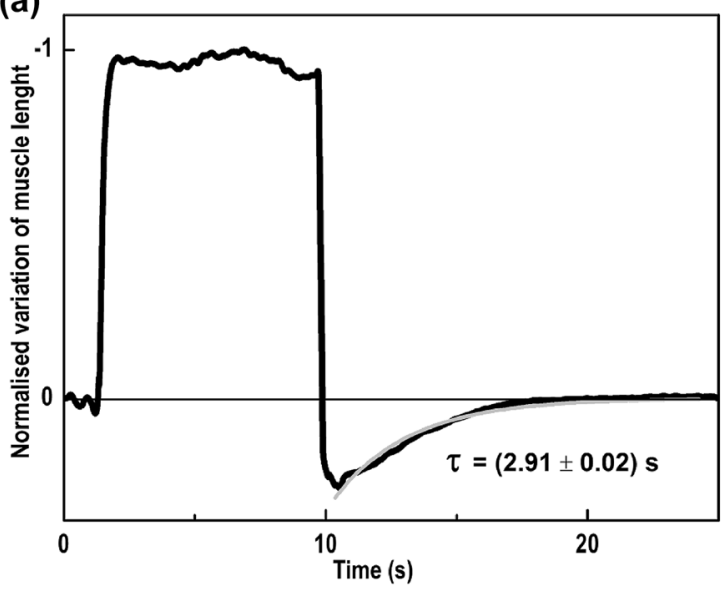

Fig. 9 a The observed muscle response for an athlete capable of delivering a relatively steady hold phase for a maximum voluntary isometric contraction of the GM is presented. b Monitoring of the (b)

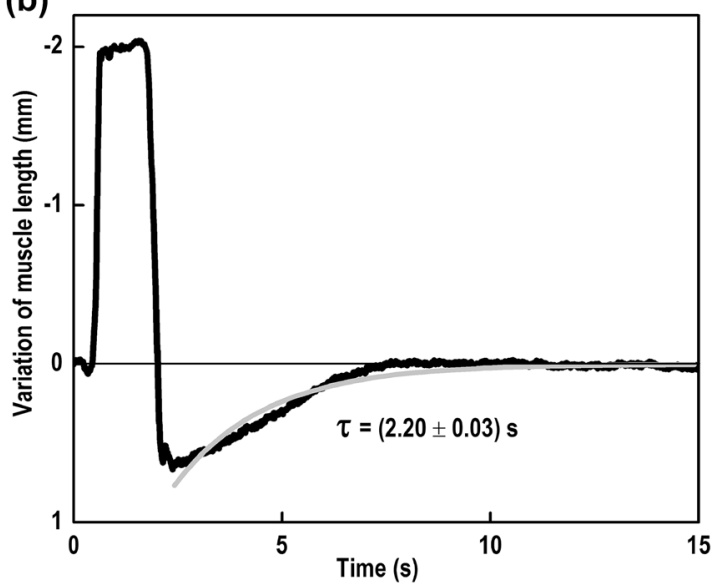

GM for isometric contraction, with the monitoring ultrasonic beam rotated by $90^{\circ}$ around the axis of the muscle with respect to a and the orientation used in all other presented results
Fig. 10 a Initial phase of the monitored muscle response (black squares) together with the normalized input (round black dots) delayed by the response time and the response (grey line) modelled with an adjusted 32nd order low-pass filter. b Finite element representation of an electrical low-pass filter of order n. c Arrangement of parallel delay lines suitable to deliver a similar response as a highorder low-pass filter
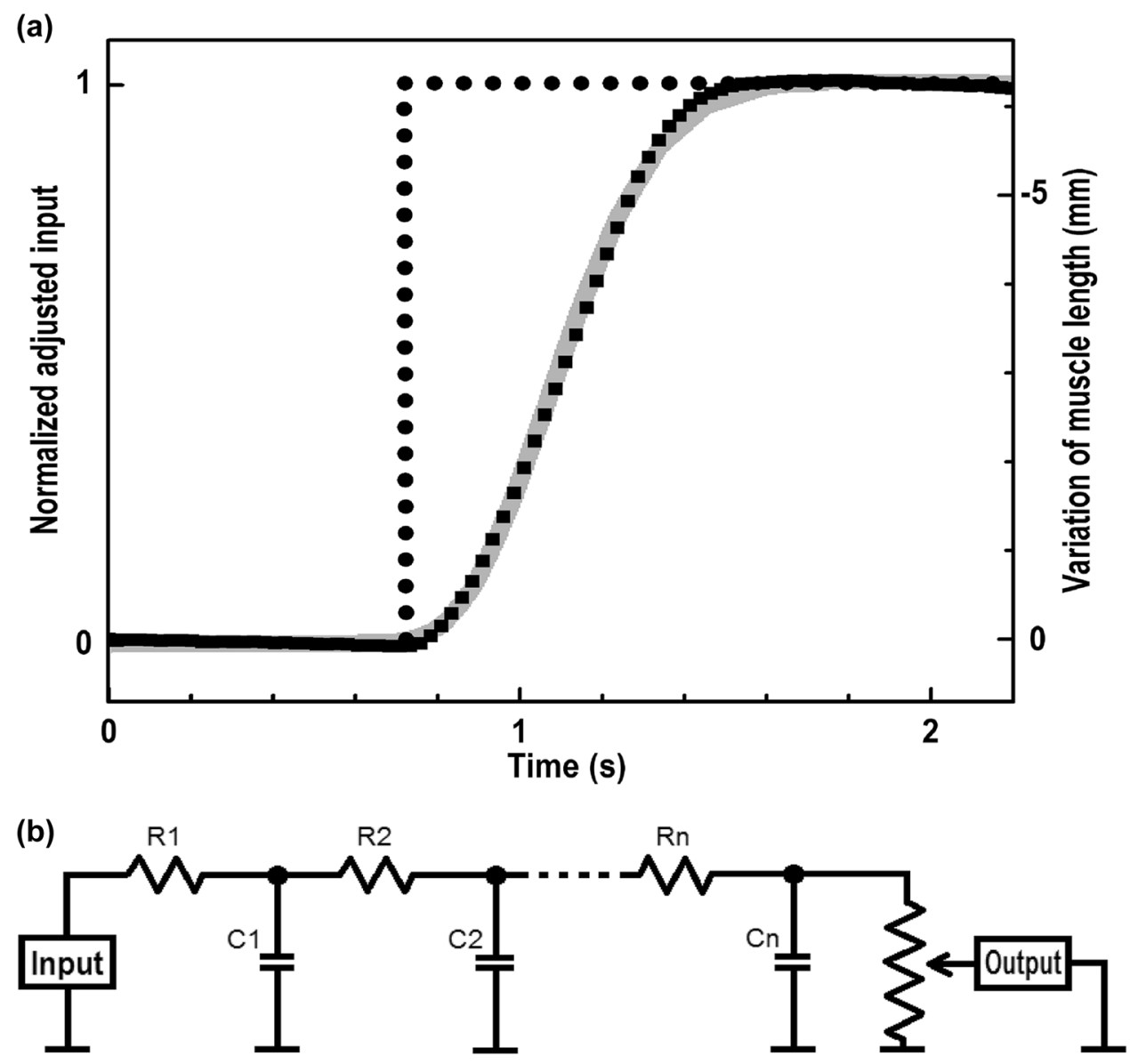

(c)

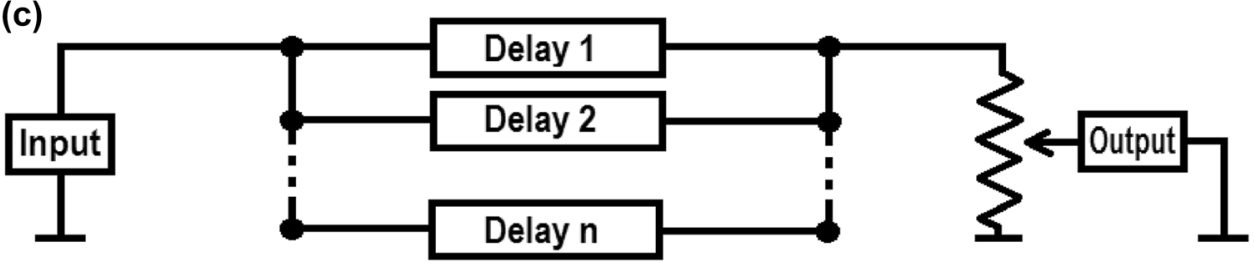


(a)

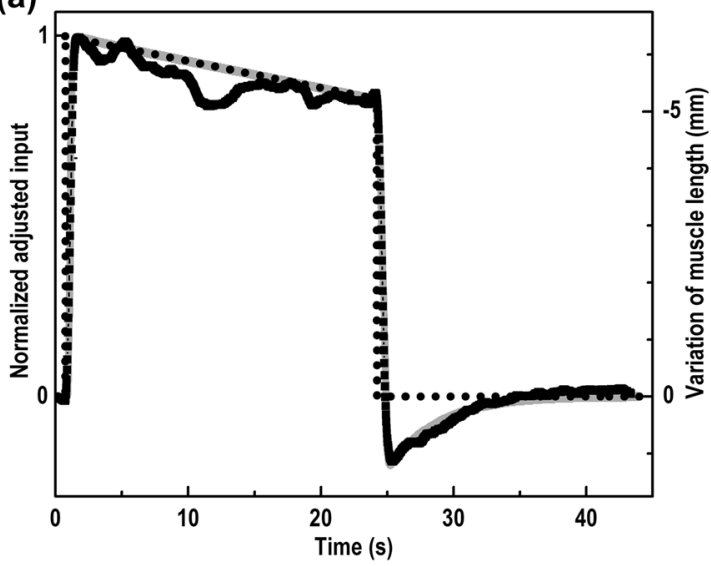

Fig. 11 a Displayed is the input (round black dots) adjusted to the response time and the droop, and height of the monitored response (black squares) together with the response (grey line) obtained for the combined electrical and mechanical lumped

of illustrating the respective features and effects happening on relatively short time scales, the data points derived from experimental monitoring are displayed on magnified scales in Fig. 10. It shows the initial rise related to activation and in Fig. 11 the rapid drop associated with deactivation. A delay of $(657.37 \pm 0.01) \mathrm{ms}$ for the onset of the muscle response from the initiated visual command given at time $t=0$ was observed for one of our subjects.

Variations of the muscle length near the initial and final inflection points of this contraction are observed to be smooth and approximately symmetric. As the nearly symmetric response is rounded of at the top and bottom, a high-order filter is required to derive the observed response from a rectangular input function. From respective simulations, it has been derived that a filter with an order of at least 30 is needed to reach a suitable output.

It appears rather unlikely though that filters of the order of at least 30 are physically present in the real nervous system. The delay from initiation of the visual signal to initiation of muscle response relies on the following: sensory perception of the signal, neural transit time to the brain, central processing, neural transit time to the muscles along the motor pathway, neuromuscular transmission, and electromechanical delay. The response caused by such filters can also be produced by parallel arranged delay elements with a suitable distribution of delay times, also called delay lines. The resulting alternative model with an equivalent kernel filter [26] is represented in Fig. 10c.

The muscle is organized into motor units where each motor neurone is connected to a number of muscle fibres. Modelling the initiation and stop of the contraction can be achieved with similar multiple units (parallel delay line filter) to obtain a slow and smooth increase or decrease

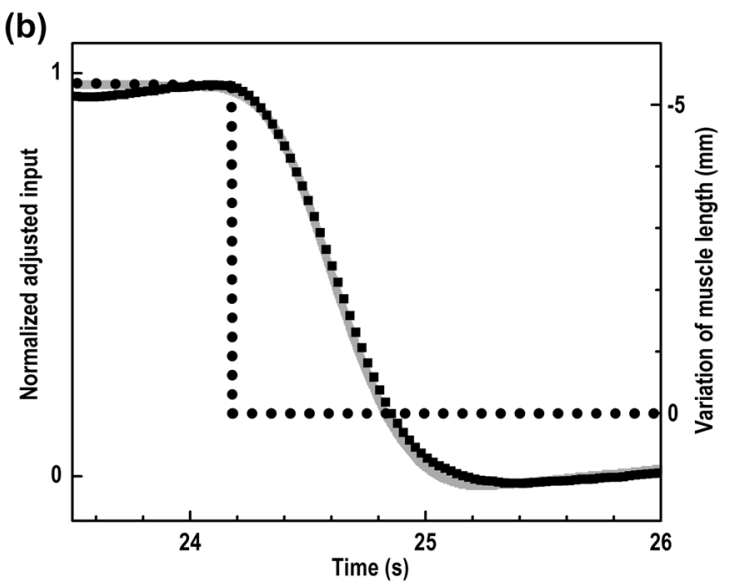

circuits with parameters adjusted to represent the monitored response in close approximation. $\mathbf{b}$ Range of rapid expansion with magnified time scale (for contraction see Fig. 10a)

in activation. The increasing and decreasing response is modelled within close approximation. A pronounced droop with irregular fluctuations was observed during the hold phase and has been attributed to local muscle fatigue. The rather elementary but unconventional modelling employing an arrangement of idealized mechanical elements including a ratchet and an adapted electronic distributed delay line filter relating to the signal transport in the nervous system, can mimic the monitored response under isometric contraction in close approximation as demonstrated in Figs. 10a, 11. Parameters (mean \pm SD) derived from the monitored muscle dynamics are listed in Table 1.

Table 1 Parameters derived from the monitored muscle dynamics (MVIC: maximum voluntary isometric contraction)

\begin{tabular}{lc}
\hline Parameters & Results \\
\hline$T(\mathrm{~ms})$ & $657 \pm 1$ \\
$C(\mathrm{~ms})$ & $958 \pm 1$ \\
Contraction speed $(\mathrm{mm} / \mathrm{s})$ & $19 \pm 0.5$ \\
$H(\mathrm{~s})$ & $22.6 \pm 0.1$ \\
Holding slope $(\mathrm{mm} / \mathrm{s})$ & $-0.07 \pm 0.01$ \\
Fluctuation $(\mathrm{mm})$ & $0.82 \pm 0.01$ \\
$R$ (ms) & $1162 \pm 2$ \\
Relaxation speed (mm/s) & $-14 \pm 0.5$ \\
Undershoot $(\%)$ & $19 \pm 0.5$ \\
Recovery time constant $\tau(\mathrm{s})$ & $4.40 \pm 0.8$ \\
Max. shortening $(\mathrm{mm})$ & $-6.36 \pm 0.12$ \\
Max. lengthening $(\mathrm{mm})$ & $7.62 \pm 0.18$ \\
\hline
\end{tabular}




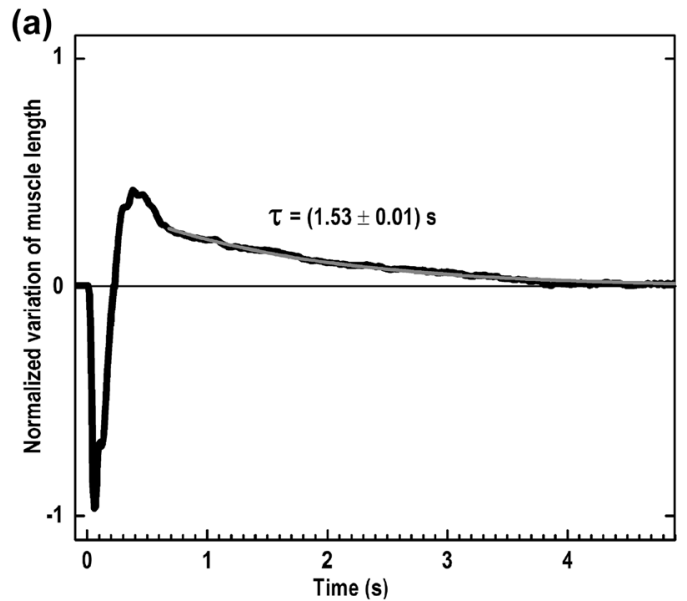

Fig. 12 Monitored response to electrical stimulation. a Extended response exhibiting a pronounced overshoot following relaxation with a final decay time constant of about $(1.53 \pm 0.01)$ s. b Electrical stimulus used for muscle activation with timing in both graphs

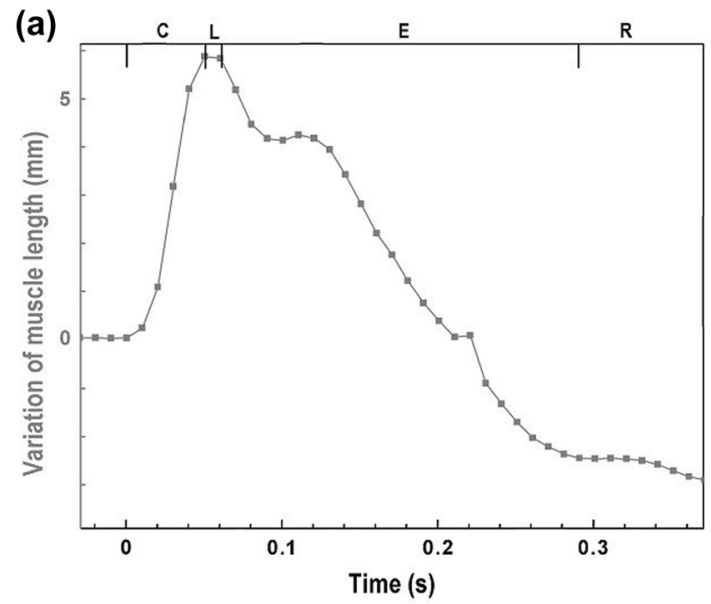

Fig. 13 a Monitored GM response to electrical stimulation. The letters relate to: $C$ : contraction phase, $L$ : latent phase, $E$ : extension phase, and $R$ : recovery phase of the muscle dynamics. $\mathbf{b}$ Rise time comparison between muscle dynamics data observed for external

\subsection{Position of the high-order filter}

Results obtained from the external electrical stimulation are shown in Fig. 12a. A typical single twitch activation followed by the monitored GM dynamics is shown here. The results of synchronous monitoring are displayed in Fig. 12b. To obtain a temporal resolution higher than the sampling time, a parabolic fit has been used to interpolate the timing for the onset of the muscle movement. The, respectively, derived latent period for the muscle movement is found to be $(4.75 \pm 0.25) \mathrm{ms}$.

The rise time observed for muscle dimension monitored by ultrasound was about $0.045 \mathrm{~s}$ (Fig. 13a) when (b)

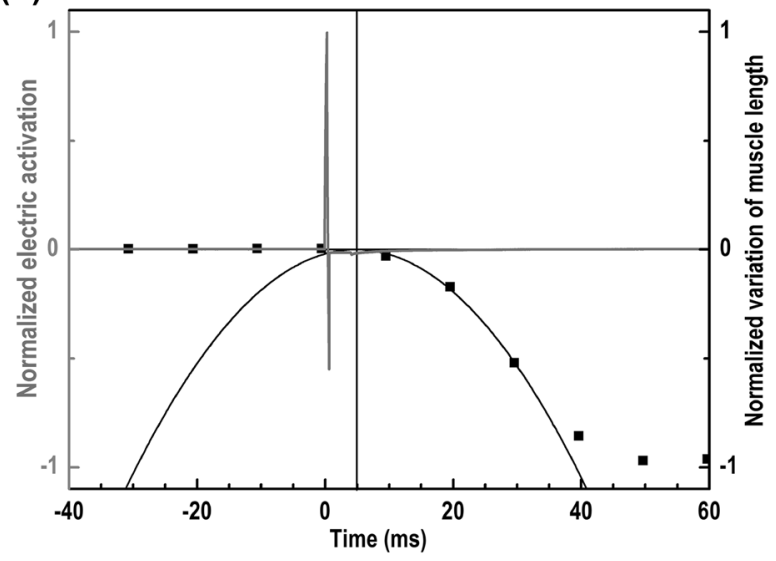

adjusted to the onset of the electrical signal (grey). The black dots represent the results of ultrasonic monitoring performed every $10 \mathrm{~ms}$ with the onset determined by a parabolic fit (black line)

(b)

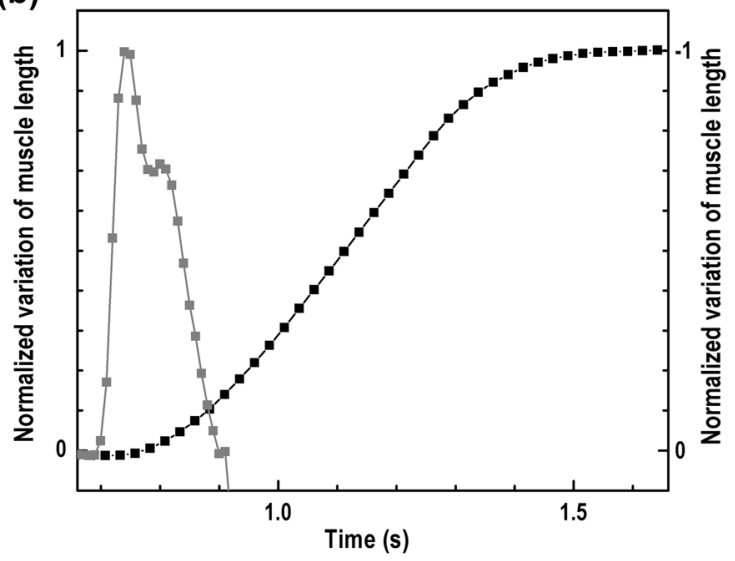

electrical stimulation (as in a; grey squares) and brain controlled activation (black squares). The time scales in both graphs are shifted to obtain 0 for the onset of contraction

the muscle contraction was elicited by external electric stimulus. For brain controlled contraction, the respective rise time was about $0.96 \mathrm{~s}$ (Fig. 13b) and therefore about 21 times longer. The magnitude of the two responses is quite similar, so the primary reason for the difference is that in the stimulated response the initial rate of change is faster than in the voluntary contraction (Fig. 13). This indicates that at least a major part of the filter function, necessary to describe the response, resides within the nervous system, which is bypassed here by the external electric stimulation of the muscle.

Nevertheless, it has to be stated that besides of the differences in involved units concerning the response, 
(a)

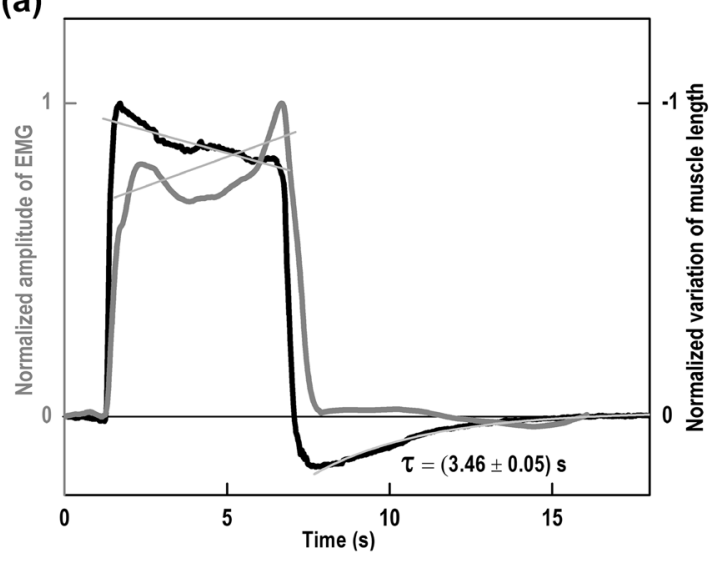

Fig. 14 a Monitoring of the dynamics of the GM for brain controlled maximum voluntary isometric contraction (black) in comparison to the simultaneously recorded normalized (integrated) EMG signal (grey). b Specific recovery time constants for the return

mechanical and electrical lumped circuits are interchangeable [21]. The two models are presented independently here to relate as closely as reasonably possible to actual active biological elements in the live muscle, the tendon, and the connected nervous system.

To support details of the modelling and to further clarify the results, additional experiments have been performed. The experimental findings of synchronously monitored muscle dynamics and EMG signals are presented in Fig. 14a. The lines fitted for the holding phase demonstrate the mentally activated correction for the drop in the muscle activity, insufficient for a full correction. The temporal resolution achieved with the EMG signals is slightly reduced with respect to that of the ultrasound monitoring.

Figure $14 \mathrm{~b}$ demonstrates that the holding phase is associated with a rising EMG signal for voluntary isometric contraction. The athlete does not reach a steady hold phase in the contractile response. The EMG signal reached an early peak value of $0.80 \pm 0.01$ then fell in the middle of the MVIC holding phase to $0.68 \pm 0.01$ before rising to a peak value of $0.99 \pm 0.01$. The corresponding values for muscle length were $0.99 \pm 0.01,0.84 \pm 0.01$, and $0.79 \pm 0.01$, respectively. This indicates that effort is maintained to achieve the demanded maximum voluntary isometric contraction and the decline in length deviation must be due to changes within the muscle.

To further quantify details related to the recovery from overshoot, the dependence of the recovery time constant on the duration of maximum voluntary isometric contraction has been determined. The results of this experiment are displayed in Fig. 14b. The athlete relating to the graph with the grey dots could not hold the longer contractions.

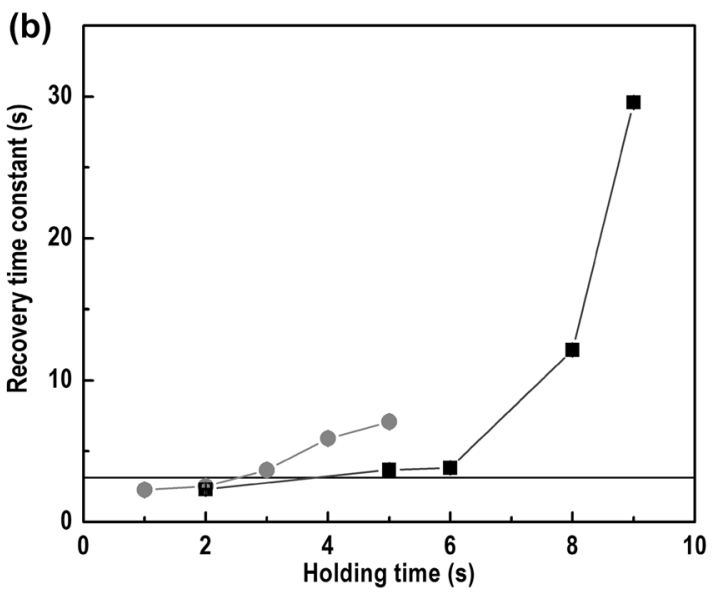

to baseline as a function of holding time for two athletes (grey dots and black squares). The horizontal black line indicates the observed asymptotic behaviour for diminishing holding times

For the second athlete's monitored activity, a rapid increase in the recovery time constant as contraction duration increased was observed for longer duration contractions until finally his maximum activity level is reached. The horizontal line indicates an averaged recovery time constant suitable for modelling of the range of holding times where strong deviations due to overload or fatigue are not present. For short holding times, up to typically 5 or $6 \mathrm{~s}$, only a slight increase in $\mathrm{t}$ is observed. A value for the recovery time constant of about $3 \mathrm{~s}$ is suitable. For the athlete able to hold a longer maximum time, a pronounced increase in the recovery time constant is observed, reaching $30 \mathrm{~s}$. This study demonstrates that the overshoot and recovery show a pronounced increase, when the limits of performance are approached.

To further quantify the overshoot and recovery, the relationship between the area of the overshoot and holding duration is displayed in Fig. 15b. A rather stable linear relationship is observed with a pronounced offset if extrapolated back to zero hold time. Since a holding time can only be accumulated once the contraction phase is achieved and since the holding phase is always followed by rapid relaxation prior to the overshoot, the observed offset for just a vanishing holding time of about $30 \%$ of the possible maximum can be attributed to these two phases.

This reasoning is supported by monitoring with external electric stimulation where even relatively short contractions exhibit the overshoot and recovery, as shown in Fig. 12a. Since in this case the overshoot and recovery are observed without the activation from the brain, the observed changes must be caused by biomechanical or physical effects within the muscle as modelled here. 

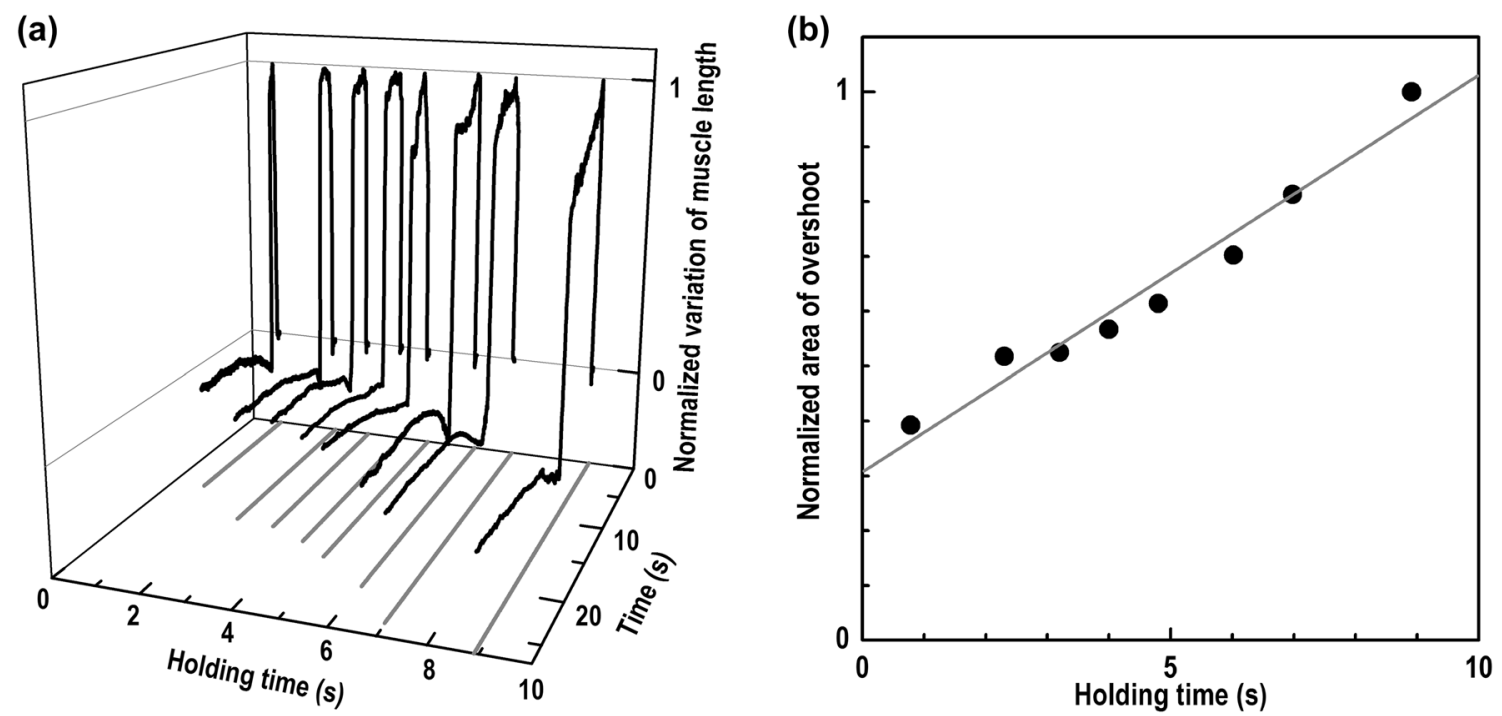

Fig. 15 a The graph shows the monitored GM dynamics for different durations of holding times for the same person on the same day. $\mathbf{b}$ The dependence of the area related to the overshoot following relaxation on the holding time (as displayed in a) together with a linear fit

\section{Discussion and conclusion}

The results of monitoring muscle dynamics with an acoustic calliper are presented together with adapted modelling using a lumped mechanical circuit consisting of springs, dampers, ratchets, and an activating linear motor drive as elements. The lumped mechanical model combined with a high-order electronic filter attributed to the nervous system is capable of modelling the observed results of voluntary isometric contractions of the GM in close approximation. Additional corrections relating to the voluntary control of the hold phase under maximum voluntary isometric contraction are introduced empirically whenever observed. The reaction time of the monitored muscle following either an external command or an electrical stimulus is introduced as additional parameter to consider. Observations based on synchronous monitoring of EMG signals as well as on direct external electric stimulation show that the overshoot and subsequent recovery are a property of the muscle. Analysis of these observations supports the modelling and additionally relates the overshoot and recovery in a quantitative manner to preceding activities of the muscle, not yet included in the presented model. The observed dependence of the overshoot and recovery on the duration of the contraction phase is left to future more refined modelling. The overshoot and recovery were observed for all athletes and, although not reported here, has additionally also been observed in the biceps brachii muscle with the same high-resolution ultrasonic calliper.

The observed overshoot and recovery described here have not previously been reported. Representing this effect required a Poynting-Thomson element with a ratchet in series with a damper for modelling. With respect to A. V. Hill's original modelling, only slight modifications regarding the bidirectional linear drive and the absence of a spring counteracting the original unidirectional drive were implemented together with an additional high-order electronic filter representing the nervous system capable of mimicking the results of in vivo measures for isometric contraction in close approximation for the contraction and hold phase. The elements additionally implemented here with respect to the original model of $A$. $V$. Hill, a ratchet in serial with a damper and both arranged in parallel with a spring, behave similar to a shock absorber. The energy transferred to such a system is effectively thermalized as well as demonstrated by any suspension of vehicles involving shock absorbers. The asymmetry caused by the ratchet acting as mechanical diode has so far neither been monitored nor been included in modelling of muscle dynamics based on lumped mechanical circuits. The influence of the novel components in modelling required by the performed monitoring is most pronounced for temporally extended muscle activities up to ultimate performance employed here for demonstration: an all-out muscle activity kept for the maximum time span possible for the performing athlete.

The earlier discussed historic catatonus experiment [18] also involves an involuntary isometric contraction but different to the conditions for the results presented here; this condition is terminated following the end of the allout contraction. Subsequently, observed is a movement of the free limb caused by the involved relaxation of the activated muscle. This movement relates to shortening of 
the muscle and exhibits a time constant of a similar order of magnitude as observed here under extended isometric conditions.

Monitoring of the skeletal muscle dynamics presented here was solely performed under isometric conditions to demonstrate previously not modelled and so far not studied effects. No movement of limbs was involved; therefore, point masses were not required for modelling with lumped mechanical elements. If moving limbs and body movements are to be considered, suitable point masses could easily be added to the developed model (Fig. 4) in a similar manner as in the model of Makssoud et al. [20] shown in Fig. 1b. Idealized elements such as springs, dampers, and linear motors are already widely used in mechanical modelling of skeletal muscles. The only additional new element required in our developed model is a mechanical ratchet. As shown with this work, it can easily be added to already established skeletal muscle models.

Some additionally observed novel effects relate to possible fatigue. They have been quantitatively demonstrated for extended tetanus (Fig. 15a) but are not included in the modelling presented here. The extension of the model to include these responses requires more refined nonlinear effects than already introduced by the implemented mechanical ratchet and is currently under study and development.

The findings reported here clearly indicate that a not previously identified nonlinear behaviour is present in muscle dynamics. Among other effects this adds a new channel for the thermalization of energy in muscles' dynamic processes. It has to be left to future studies to relate the experimentally observed and modelled effects to the three-dimensionality of the real muscle. Additional research is also needed to refine the physiological role of the here presented nonlinear effect in muscle contraction and relaxation. In that respect it has been identified that the nonlinear element relates to biomechanical components of the muscle and cannot be attributed to the nervous system.

Acknowledgements Some of the signal processing and data evaluation software were developed with support of the European Commission under the European Union 7th Framework Program within AISHAIl (Aircraft Integrated Structural Health Assessment II, EU-FP7CP 212912). Furthermore, helpful discussions with Brian Maclntosh, Human Performance Laboratory of the University of Calgary, are gratefully appreciated and acknowledged.

\section{Compliance with ethical standards}

Conflict of interest Hereby, we state that we have submitted our work as an original article and have made substantial contributions to the following: The conception and design of the study, data acquisition, analysis and interpretation of data, drafting the article or revising it critically for important intellectual content. We have read and concur with the content in the manuscript, and the final version is submitted with the approval of all authors. We do not have any financial or personal relationships with other people or organizations that could inappropriately influence or bias our work. The material within this manuscript has not been and will not be submitted for publication elsewhere except as an abstract.

Ethical standards In all cases presented, human studies have been approved by the appropriate ethics committee according to requirements and have therefore been performed in accordance with the ethical standards laid down in the 1964 Declaration of Helsinki. All persons involved in monitoring gave their informed consent prior to their inclusion in the study.

\section{References}

1. Abramowitz M, Irene A, Stegun Ed (1972) Handbook of mathematical functions with formulas, graphs, and mathematical tables. National Bureau of Standards. Applied Mathematics Series-55, The Superintendent of Documents, Washington, D.C. U.S. Government Printing Office

2. Baskin RJ, Paolini PJ (1967) Volume change and pressure development in muscle during contraction. Am J Physiol 213:1025-1030

3. Cross Robert A (2006) Myosin's mechanical ratchet. Proc Natl Acad Sci 103(24):8911-8912. https://doi.org/10.1073/ pnas.0603213103

4. Elliott GF, Lowy J, Worthington CR (1967) Low-angle X-ray diffraction from living striated muscle during contraction. J Mol Biol 6:295-305

5. Forbes A, McH Baird PC, Hopkins A (1926) The involuntary contraction following isometric contraction of skeletal muscle in man. Am J Physiol 78(1):81-103

6. Fung YC (1981) Biomechanics: mechanical properties of living tissues. Springer, New York. ISBN 0-387-90472-7

7. Hill AV (1938) The heat of shortening and the dynamic constants of muscle. Proc R Soc Ser B126:136-195

8. Hill AV (1951) The earliest manifestation of the mechanical response of striated muscle. Proc R Soc Ser B Biol Sci 138:339-348

9. Hossain MZ, Grill W (2019) High-resolution monitoring of the velocity of ultrasound in contracted and relaxed human muscle. Comput Methods Biomech Biomed Eng Imaging Vis. https://doi. org/10.1080/21681163.2018.1449138

10. Hossain $M Z$, Grill W (2012) Noninvasive ultrasonic monitoring of the mechanical properties of selected muscles and connected tendons. In: Health monitoring of structural and biological systems, San Diego, vol 8348, p 83480U. https://doi. org/10.1117/12.914978

11. Hossain MZ, Grill W (2011) Synchronous monitoring of muscle dynamics and electromyogram. In: Health monitoring of structural and biological systems, San Diego, vol 7984, p 798419. https://doi.org/10.1117/12.880154

12. Hossain MZ (2012) High resolution ultrasonic monitoring of muscle dynamics and novel approach to modelling. Thesis, Chapter 2-3. http://www.qucosa.de/recherche/frontdoor/?tx_ slubo pus4frontend\%5Bid\%5D=urn:nbn:de:bsz:15-qucos a-102502

13. Hossain MZ, Twerdowski E, Grill W (2008) High speed ultrasound monitoring in the field of sports biomechanics. In: Proceedings of SPIE health monitoring of structural and biological systems, San Diego, vol 6935, pp 70-78. https://doi. org/10.1117/12.776111 
14. Hossain MZ, Voigt $H$, Grill W (2009) Monitoring of variations in the speed of sound in contracting and relaxing muscle. In: Proceedings of SPIE health monitoring of structural and biological systems, San Diego, vol 7295. https://doi.org/10.1117/12.81203 6

15. Huijing PA (1995) Parameter interdependence and success of skeletal muscle modelling. Hum Mov Sci 14:443-486. https:// doi.org/10.1016/0167-9457(95)00024-8

16. Huxley HE, Brown W (1967) Low angle X-ray diagram of vertebrate striated muscle and its behaviour during contraction and rigor. J Mol Biol 30:383-434

17. Kardel T (1994) Steno on muscles, introductions, texts, translations. Trans Am Philos Soc 84:1-252

18. Kohnstamm O (1915) Demonstration einer katatonieartigen Erscheinung beim Gesunden (Katatonusversuch). Neurologisches Zentralblatt 34:290-291

19. Linden BJJJ, Koopman HFJM, Grootenboer HJ, Huijing PA (1998) Modeling functional effects of muscle geometry. J Electromyog Kinesiol 8:101-109

20. Makssoud HEL, Guiraud D, Poignet P (2004) Enhancement of physiological and mechanical modelling of the skeletal muscle controlled by functional electrical stimulation. In: Proceedings of 9th annual conference of the international FES Society, Bournemouth. https://pdfs.semanticscholar.org/fa5c/1ea8d c1a8f0dc4cf7f4c6e49039dde0739f9.pdf

21. Martini R, Frederic H (2005) Fundamentals of anatomy and physiology, 7th edn. Pearson Education, New Jersey

22. Mason FM, Tworoger M, Martin AC (2013) Apical domain polarization localizes actin-myosin activity to drive ratchet-like apical constriction. Nat Cell Biol 15(8):926-942. https://doi. org/10.1038/ncb2796

23. Matsubara I, Elliott GI (1962) X-ray diffraction studies on skinned single fibres of frog skeletal muscle. J Mol Biol 72:657-669

24. Rome E (1967) Light and X-ray diffraction studies of the filament lattice of glycerol-extracted rabbit psoas muscle. J Mol Biol 27:591-602

25. Salmon A (1916) D'un intéressant phénomène d'automatisme qu'on remarque après les efforts musculaires chezles sujets sains. Revue Neurologique 23:27-34

26. Sayed HA (2003) Fundamentals of adaptive filtering. Wiley, New Jersey

27. Sherwood L (2010) Human physiology: from cells to systems, muscle physiology, 7th edn. Cengage Learning, Belmont, pp 257-268

28. Swammerdam J, Biblia Naturae Leiden (1737) Cited from Kardel, 1994

29. Yamaguchi TG (2001) Dynamic modelling of musculoskeletal motion: a vectorized approach for biochemical analysis in three dimensions. Springer, New York, p 233

30. Zienkiewicz OC, Taylor RL (1991) The finite element method, vol 2. McGraw-Hill Book Company, London

31. Zuurbier CJ, Huijing PA (1992) Influence of muscle geometry on shortening speed of fiber, aponeurosis and muscle. J Biomech 25:1017-1026

Publisher's Note Springer Nature remains neutral with regard to jurisdictional claims in published maps and institutional affiliations. 